OPEN ACCESS

Edited by:

Miao Yu,

Chongqing University, China

Reviewed by:

Rongjia Tao,

Temple University, United States

Jie Zhang,

Chongqing University, China

*Correspondence:

Ubaidillah

ubaidillah_ft@staff.uns.ac.id Seung-Bok Choi

seungbok@inha.ac.kr

Saiful Amri Mazlan

amri.k@utm.my

Specialty section:

This article was submitted to

Smart Materials,

a section of the journal

Frontiers in Materials

Received: 27 November 2018 Accepted: 27 February 2019

Published: 26 March 2019

Citation:

Ubaidillah, Purnomo ED, Ismail $H$,

Choi S-B, Aziz AA and Mazlan SA

(2019) Swelling, Thermal, and Shear

Properties of a Waste Tire Rubber Based Magnetorheological Elastomer.

Front. Mater. 6:47.

doi: 10.3389/fmats.2019.00047

\section{Swelling, Thermal, and Shear Properties of a Waste Tire Rubber Based Magnetorheological Elastomer}

\author{
Ubaidillah $^{1 *}$, Endra Dwi Purnomo ${ }^{1}$, Hanafi Ismail ${ }^{2}$, Seung-Bok Choi ${ }^{3 *}$, Aishah Abdul Aziz ${ }^{4}$ \\ and Saiful Amri Mazlan ${ }^{4 *}$
}

${ }^{1}$ Fungsional Materials and Actuators Laboratory, Mechanical Engineering Department, Universitas Sebelas Maret, Surakarta, Indonesia, ${ }^{2}$ School of Materials and Mineral Resources Engineering, USM Engineering, Nibong Tebal, Malaysia, ${ }^{3}$ Department of Mechanical Engineering, Inha University, Incheon, South Korea, ${ }^{4}$ Engineering Materials and Structures (eMast) iKohza, Malaysia Japan International Institute of Technology (MJIIT), Universiti Teknologi Malaysia, Kuala Lumpur, Malaysia

In this study, the achievement of revulcanization, thermal stability, and rheological behaviors of waste tire rubber (WTR), based magnetorheological elastomers (MREs), were evaluated to convince their applicability as one of smart material possessing the field-dependent tuning capability of material characteristics such as complex modulus. The cross-linking density of the MREs was assessed through the swelling test to ratify the degree of reclaiming. The behavior of MREs to the temperature enhancement was evaluated via thermogravimetric analysis and thermomechanical analysis. Meanwhile, the alteration of shear stress depending on the magnetic fields was investigated at both steady and oscillatory states, measured using a rheometer. It has been shown that the increment of shear stress is proportional to the increment of the magnetic field. More specifically, it has been found that the WTR based MRE can achieve the maximum static stress ranging from 9 to $13 \mathrm{kPa}$ (at $656 \mathrm{mT}$ ) with a linear viscoelastic (LVE) region above $3 \%$ strain amplitude. In addition, from the oscillatory test it has been determined that the highest MR effect of $24.71 \%$ can be achieved, which directly indicates the augmentation of both storage and loss moduli under ramped frequency and strain. Finally, it has been shown that the highest degree of reclamation based on swelling test can be achieved up to $54 \%$, confirming the occurrence of the crosslinking during the reclamation process.

Keywords: waste tire rubber, magnetorheological elastomer, thermal analysis, swelling test, MR effect

\section{INTRODUCTION}

Reclaiming waste tire is usually performed by blending waste tire rubber (WTR) to the virgin rubber or revulcanization the inert rubber by treating them in physical and chemical ways. Reclamation of rubber is mainly scission or cleavage of sulfur-sulfur, carbon-sulfur, or carbon-carbon to break down three-dimensional network structures from the vulcanization process. At the same time, the division bonds are reformed through a revulcanization mechanism obtaining new rubber products. Application of temperature, shear force and chemical agents could facilitate such physical processes (Tripathy et al., 2002) including screw extruder (Formela et al., 2014; Piszczyk et al., 2014), roll mill-compression molding (De et al., 2013), internal mixer $[5,6,7]$, and high-pressure high-temperature (HPHT) sintering reclamation techniques (Morin et al., 2002; Tripathy et al., 2002). 
So far, recycled rubber-based products exhibit non-tunable mechanical properties. The WTR reclamation would draw attentions when the reclaimed articles have smart material characteristics. Here, smart features refers to the capability of goods reacting to the external condition in which smart materials only own the behavior. Such capability could enhance the economic value of the recycled product. According to the advancement of rubber reclaiming technologies, waste tire rubber has the potential to be reclaimed as smart materials, namely, magnetorheological elastomers (MREs). MREs have taken a prominent position in actively tunable vibration devices such as automotive bushing, rotating machine mounting and a seismic damper, due to their tunable properties by the intensity of the magnetic fields ( $\mathrm{Li}$ et al., 2014; Ubaidillah et al., 2015). MREs are also compatible with the sensory application and morphing structure devices (Ottaviani et al., 2005; Li et al., 2009; Ghafoorianfar et al., 2013; Ausanio et al., 2014; Bica et al., 2014).

Based on the survey provided by Ubaidillah et al. (2015), all scientific reports published since the 1990s proposed MREs using virgin elastomers in which the vulcanization mechanism did not become the main issue. And so far, most of the research interests have been addressed on the enhancing rheological properties such as MR effect, storing, and dissipating energy performances. Some efforts were carried out for enhancing MREs properties such as; (a) manipulating the matrix, either by using single type matrix or mixed matrix, (b) controlling the particle types, size, morphology, and composition, and (c) manipulating cross-link density through operating additives and processing condition. The latest research relating to the vulcanizing issue was undertaken by considering self-crosslinking between two different compounds. Wang et al. (2015) fabricated new class MREs by blending polychloroprene rubber with epoxidized natural rubber, without curing agents that the so-called self-cross-linking MREs. This work indeed provided a breakthrough for the MREs preparation since there was no additive involvement in the vulcanization process. Again, the self-cross-linked MREs preparation incorporated virgin rubber. Therefore, the MREs fabrication based on waste tire rubber would be a fascinating exploration of some aspects in terms of manufacturing and performance.

Regarding to the challenges, Ubaidillah et al. (2016a,b,c) have successfully reclaimed WTR as the primary matrix of MREs. First, Ubaidillah et al. (2016c) fabricated MREs utilizing WTR as the matrix as well as $\mathrm{Fe} 3 \mathrm{O} 4$ (magnetite) powders obtained from electronic wastes. In this work, the specimen's physicochemical properties and stress-strain behaviors were evaluated through the static compression test. Pursuing the complete investigation on dynamic compression properties, the magneto-induced rheological properties of the samples were determined as can be found in Ubaidillah et al. (2016b). It became evident that the MR effect of WTR based MREs featuring magnetite achieved about 15\% MR effect under dynamic compression loadings. To enhance the magnetic properties, the magnetite powders were then replaced with carbonyl iron (CI) particles. As reported by Ubaidillah et al. (2016a), WTR based MREs were examined for their physicochemical and rheological properties under dynamic shear loadings. The MREs achieved a higher MR effect that was about $24.71 \%$. As surveyed from the literatures Ubaidillah et al. (2016a,b,c), both swelling problem and thermal analysis have not yet been treated, even though these are important issues to be addressed in order to ensure high stability and reliability of the field-dependent rheological properties of the WTR based MREs.

Consequently, the technical originality of this work was to investigate the swelling, thermal, steady state, and dynamic properties of the WTR based MREs. This study is important as

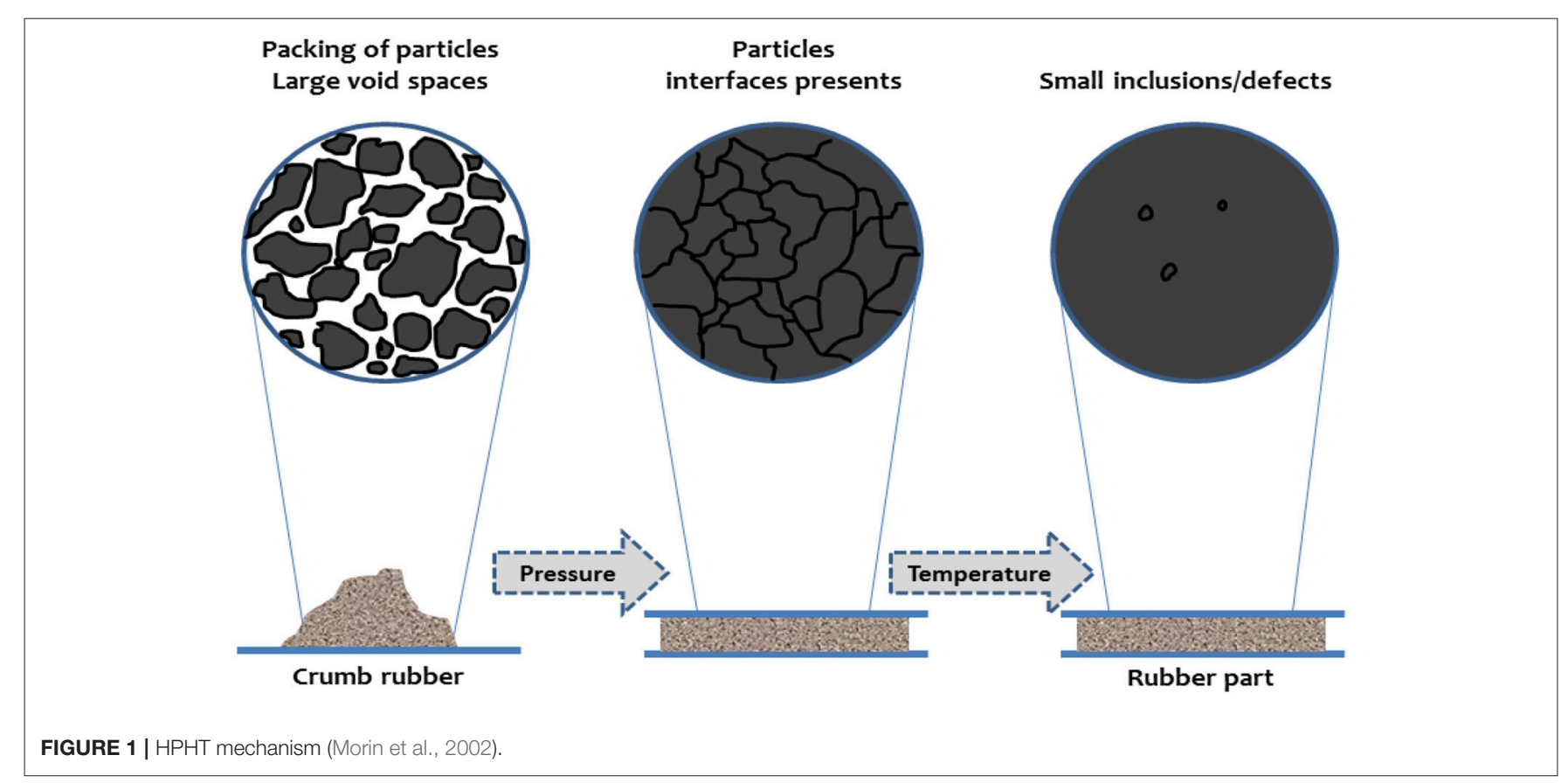


it is necessary to determine why the hesitation on the reliability issue, since it is known that the waste rubber is hard to be reclaimed without incorporating virgin rubber. Swelling tests are first conducted to ensure the revulcanization process within MREs based on the obtained swelling parameters. Thermal analyzes are provided to study the stability of the synthesized samples. The properties, such as the dependence magnetic fields, are explored through the rheological test in both absence and presence of magnetic fields in the shear direction. The steady state loading reveals the stress-strain properties of waste tire based MREs including understanding in the linear viscoelastic region. Authors believe that the utilization of the WTR for raw materials of the MREs is a useful idea for waste tire management and a clean environment.

\section{MATERIALS AND RECLAMATION PROCESS}

The company of PT Bengawan Sumber Baru, Indonesia, provided the WTR with the average size of $\approx 2.8 \mathrm{~mm}$. It was obtained from discarded tires (passenger and commercial vehicles) which were ground mechanically and free from metals and textiles. Screening was then carried out to get the uniform size of WTR (about mesh 60 ). The additives employed were chosen based on the typical rubber reclamation process such as stearic acid (98\%, Justus Kimia Raya, Indonesia), zinc oxide (99\%, $<1 \mu \mathrm{m}$, Bratachem, Indonesia), sulfur (Bratachem, Indonesia). The magnetizable particles were carbonyl iron (CI) particles, having an average size of $6 \mu \mathrm{m}$, type CN supplied by BASF, Germany. The additional latex solution was used as first raw material bonding between $\mathrm{CI}$ particles and WTR.

The HPHT sintering method implements the same principle concepts with the mechanical approach in that they both apply high temperature and high pressure. Figure 1 depicts the schematic mechanism of the HPHT sintering process. The HPHT is conducted using simultaneous high temperature (between 200 and $240^{\circ} \mathrm{C}$ ) and high-pressure compaction (above $20 \mathrm{MPa}$ ) on the ground tire rubber in a mold for a specified duration. Therefore, the process does not involve the twin screw extruder or roll mill that operates through shearing the waste rubber at a temperature of $\pm 180^{\circ} \mathrm{C}$. In this process, the cleavage and reforming of the rubber vulcanizates happen in a single step process. According to the working principle of the HPHT sintering process, the recycling procedure is simpler than that of the common mechanical approach.

The MRE samples were prepared through two major steps: mixing and reclamation. First, the WTR (100 phr) and additives (1.5 phr stearic acid, 5 phr $\mathrm{ZnO}$, and 2 phr sulfur) were mixed in a laboratory mechanical stirrer at $250 \mathrm{rpm}$ until the mixture was visually homogenous. Unlike the virgin rubber-based MREs that require a roll mill for compounding, homogenous mixtures were easily achieved since all raw materials were in powdered form except the latex solutions. The CI particles in contents of 10, 20, 30 , and $40 \mathrm{wt} \%$ were then mixed homogeneously with the WTR mixtures using a mechanical stirrer. Hereafter, the $10 \mathrm{phr}$ latex solution was added to the mixed raw materials and then further assorted until well blended. The formulation of WTR based MRE was detailed in Table $\mathbf{1 .}$

Second, the reclamation process was conducted using the laboratory HPHT sintering device which could exert 20 tons of hydraulic pressure (Krisbow, Indonesia) and was equipped with a heat controller. The molding kit was fabricated using the conventional machine and was made of mild steels. The thickness could be adjusted based on the amount of molded mixtures. Two heater cartridges were equipped with the mold having heat power of $150 \mathrm{~W}$ each. Heater controller was constructed of current driver, display monitor, K-type thermocouple, and circuit breaker. The samples were fabricated in a mold with a bore diameter of $30 \mathrm{~mm}$. The final mixtures were placed in the cavity, and the hydraulic pressure of $25 \mathrm{MPa}$ was applied at a temperature of $200^{\circ} \mathrm{C}$ for $1 \mathrm{~h}$ to accomplish revulcanization. This procedure adopted the reclamation technique by Morin et al. (2002) and Tripathy et al. (2002). The heating rate from ambient to a steady temperature was $10^{\circ} \mathrm{C} / \mathrm{min}$. Following the revulcanization process, the heater controller was turned off, and the mold was then allowed to cool until reaching ambient temperature.

\section{CHARACTERIZATION}

\section{Morphological Examination}

Field emission scanning electron microscopy (FESEM), SUPRATM 35SV, ZEISS, USA, was utilized for capturing the morphology of CI particles and their distribution within the MRE matrix. The device was operated at an accelerating voltage of $10 \mathrm{kV}$. During observation, the MRE samples were

TABLE 1 | Formulation of WTR based MRE.

\begin{tabular}{lcccc}
\hline $\begin{array}{l}\text { Compounding } \\
\text { ingredients }\end{array}$ & \multicolumn{5}{c}{ Batch weights } \\
\cline { 2 - 5 } & $\begin{array}{l}\text { MRE 10 wt\% } \\
\text { Cl particle }\end{array}$ & $\begin{array}{c}\text { MRE 20 wt\% } \\
\text { Cl particle }\end{array}$ & $\begin{array}{c}\text { MRE } \mathbf{3 0} \text { wt } \% \\
\text { CI particle }\end{array}$ & $\begin{array}{c}\text { MRE } \\
\text { Cl particle }\end{array}$ \\
\hline WTR (phr) & 100 & 100 & 100 & 100 \\
Zinc oxide (phr) & 5 & 5 & 5 & 5 \\
Stearic acid (phr) & 1.5 & 1.5 & 1.5 & 1.5 \\
Sulfur (phr) & 2 & 2 & 2 & 2 \\
Latex (phr) & 10 & 10 & 10 & 10 \\
Cl particle (wt\%) & 10 & 20 & 30 & 40
\end{tabular}

TABLE 2 | Apparent flux densities corresponding to the applied current.

\begin{tabular}{lccccc}
\hline MREs & \multicolumn{5}{c}{ Flux density (T) } \\
\cline { 2 - 6 } (Cl Particle wt\%) & 0 A & 1 A & 2 A & 3 A & 4 A \\
\hline 10 & 0 & 0.158 & 0.313 & 0.474 & 0.617 \\
20 & 0 & 0.162 & 0.320 & 0.485 & 0.633 \\
30 & 0 & 0.165 & 0.331 & 0.496 & 0.645 \\
40 & 0 & 0.169 & 0.337 & 0.505 & 0.656
\end{tabular}


crushed using a hammer after immersion in liquid nitrogen. The observed surfaces were coated with a thin layer of gold.

\section{Swelling Test}

The swelling test was conducted to examine the degree of revulcanization process of the MREs. The synthesized sample was subjected to an equilibrium swelling in toluene at ambient temperature. Small sliced MREs were initially weighed $\left(m_{1}\right)$ and then immersed in $100 \mathrm{ml}$ toluene and allowed to swell for $72 \mathrm{~h}$. The sample was then wiped to remove the toluene on its surfaces and quickly weighed to measure the swollen weight $\left(\mathrm{m}^{2}\right)$. The step was followed by drying in an oven at $80^{\circ} \mathrm{C}$ to remove small molecular weight substance until reaching same dried weight $\left(m_{3}\right)$.

The swelling parameters such as soluble fraction, swelling degree, cross-link density, and the degree of reclaiming were determined based on the recorded weight. The following relation (Equation 1) is useful for determining soluble fraction (SF),

$$
S F=\frac{m_{1}-m_{3}}{m_{1}} \times 100 \%
$$

The swelling degree $(Q)$ was calculated in accordance with Equation (2) as follows,

$$
Q=\frac{m_{2}-m_{1}}{m_{1}} \times 100 \%
$$

Meanwhile, the cross-link density of the MREs was determined based on ASTM D 6,814 that according to the Flory-Rehner formula (Flory and Rehner, 1943) without Kraus correction (Equation 3),

$$
v=\frac{-\left[\ln \left(1-V_{r}\right)+V_{r}+\chi V_{r}^{2}\right]}{\left[\frac{V_{1}\left(V_{r}^{\frac{1}{3}}-V_{r}\right)}{2}\right]}
$$

where: $v$-cross-link density, $\mathrm{mol} / \mathrm{cm}^{3} ; V_{r}$-represents volume fraction in the swollen sample; $V_{1}$-molar volume of toluene $\left(106.2 \mathrm{~cm}^{3} / \mathrm{mol}\right)$ (Yazdani et al., 2011); $\chi$-parameter of rubbersolvent interaction (in calculation, it was assumed to be 0.391 ; Formela et al., 2014).

The volume fraction of rubber was calculated according to Equation (4),

$$
V_{r}=\frac{\frac{m_{e}}{\rho_{e}}}{\frac{m_{e}}{\rho_{e}}+\frac{m_{t}}{\rho_{t}}}
$$

where: $m_{e}$-mass of dry MREs in $\operatorname{gram}(g) ; \rho_{e}$-density of dry MREs in $\frac{g}{\mathrm{~cm}^{3}} ; m_{t}$-mass of absorbed toluene by MREs in gram $(g)$; $\rho_{t}$-density of toluene in $\frac{g}{\mathrm{~cm}^{3}}$.
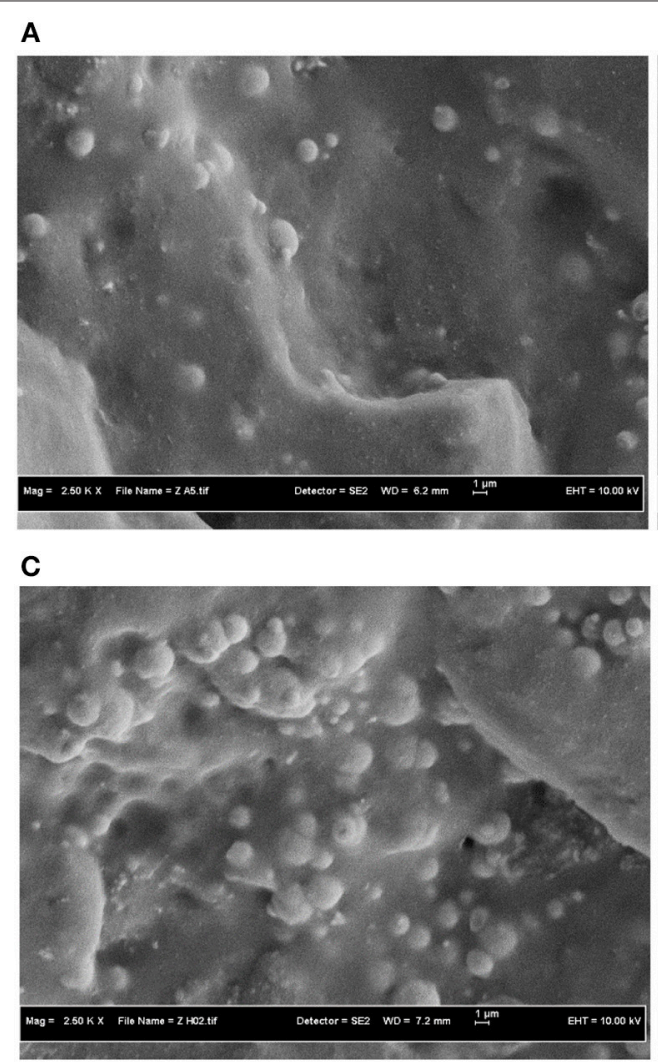

\section{B}

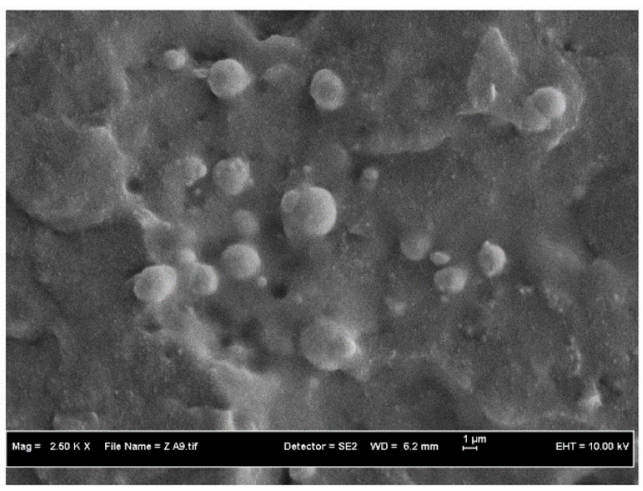

D

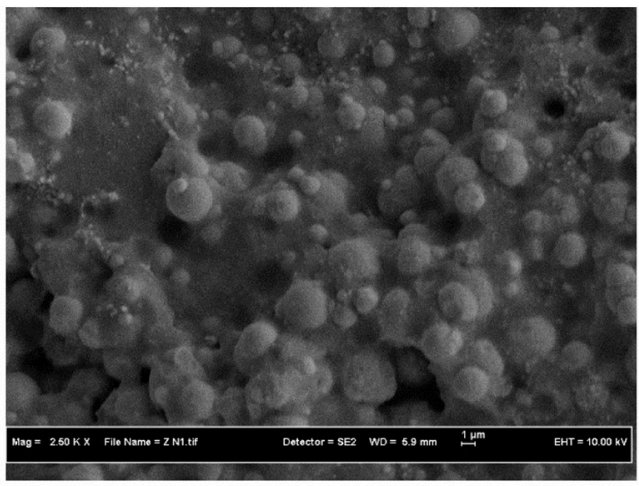

FIGURE 2 | SEM micrograph of the WTR based MRE with Cl particles: (A) 10 wt\%, (B) 20 wt\%, (C) 30 wt\%, and (D) 40 wt\%. 
The achievement of revulcanization process through HPHT sintering was determined on the basis of alteration in the crosslinking density of the reclaimed MREs $\left(v_{1}\right)$ relative to the ground tire rubber without reclamation $\left(v_{0}\right)$, regarding Equation 5 as follows,

$$
\text { Degree of reclaiming }=\frac{v_{0}-v_{1}}{v_{0}} \times 100 \%
$$

\section{Thermal Test}

The thermogravimetric tests were undertaken using TGA Q50, TA Instruments (US). The test samples had masses of 5-10 mg, and the temperature was varied from 30 to $600^{\circ} \mathrm{C}$. The heating rate was $20^{\circ} \mathrm{C} / \mathrm{min}$ under a nitrogen gas environment (100 $\mathrm{ml} / \mathrm{min}$ flow rate). The thermal expansion of MREs was evaluated using TMA Q400 TA Instruments (US). An expansion probe was equipped to measure the dimension change of an MRE sample that measured $5 \times 5 \times 10 \mathrm{~mm}$. Data were collected at a heating rate of $10^{\circ} \mathrm{C} / \mathrm{min}$ from an ambient temperature of $150^{\circ} \mathrm{C}$ while the initial load was set to $0.05 \mathrm{~N}$.

\section{Rheological Test}

Viscoelastic properties of the WTR based MREs were witnessed in a rotational shear mode using the parallel-plate rheometer (model Physica MCR 302, Anton Paar, Austria) equipped with a magnetorheological device MRD 70/1T. The diameter of parallel plate disk was $20 \mathrm{~mm}$ (PP20/MRD/T1/P2). A temperature control Viscotherm VT2, Anton Paar was also utilized for maintaining the desired testing temperature of $25^{\circ} \mathrm{C}$. The rheological measurement system with parallel-plate is described in standards ISO 6721-10 and DIN 53019-1 (Sorokin et al., 2015). An electromagnet unit in MRD 70/1T can generate a homogeneous magnetic field perpendicular to the sample and non-magnetic measurement plate. The variation of magnetic flux could be from 0 until $690 \mathrm{mT}$ by applying current from 0 to 4 A. Table 2 exhibits how the flux density alters with the current which means the flux density across the MREs samples would be different as the consequences of CI particles fraction.

Under steady condition test, the MREs sample was sheared at a constant shear rate of $100 \mathrm{~s}^{-1}$ at a zero magnetic field for $30 \mathrm{~s}$. The pre-conditioning was objected to making a uniform distribution of particle within the sample. The step was also

TABLE 3 | Swelling properties of WTR based MRE.

\begin{tabular}{lcccc}
\hline Sample & $\begin{array}{c}\text { Soluble } \\
\text { fraction (\%) }\end{array}$ & $\begin{array}{c}\text { Swelling } \\
\text { degree (\%) }\end{array}$ & $\begin{array}{c}\text { Crosslink } \\
\text { density } \\
\mathbf{( 1 0}^{-\mathbf{4}} \mathbf{~ m o l} / \mathbf{c m}^{\mathbf{3}} \mathbf{)}\end{array}$ & $\begin{array}{c}\text { Degree of } \\
\text { reclaiming } \\
\mathbf{( \% )}\end{array}$ \\
\hline Reclaimed WTR & 7.967 & 195.330 & 8.390 & 54.007 \\
MREs 10 wt\% CIP & 6.060 & 165.343 & 8.770 & 51.922 \\
MREs 20 wt\% CIP & 5.677 & 135.998 & 8.780 & 56.916 \\
MREs 30 wt\% CIP & 5.238 & 117.043 & 8.810 & 52.143 \\
MREs 40 wt\% CIP & 4.901 & 97.962 & 9.040 & 50.478 \\
THE PREVIOUS STUDY BENCHMARKING & & \\
Formela et al., 2014 & $7.4-9.6$ & NA & $3.76-7.94$ & $41-52$ \\
Yazdani et al., 2011 & NA & NA & $1.3-6.98$ & NA
\end{tabular}

aimed at releasing the possible stored residual stress. Then, it was followed by preliminary magnetic energizing for $30 \mathrm{~s}$ to make a homogeneous static attraction between iron particles within the sample. After running the preconditioning step, the sequence of steady testing was allowed to proceed. The steadystate measurement was then applied by twisting the sample in a constant strain swept with a very low strain rate. The treatment was nearly similar to a static shear test.

The magneto-induced dynamic properties of the samples were evaluated through the oscillatory test. The storage modulus changes which can be represented by the effect of ramped magnetic flux was assessed under strain amplitude of $1 \%$, and excitation frequency of $1 \mathrm{~Hz}$. The ramp magnetic flux was realized by driving an electric current from 0 to $5 \mathrm{~A}$ in a linear fashion. Moreover, the frequency swept tests from 0.1 to $100 \mathrm{~Hz}$ were carried out under a constant strain amplitude of $1 \%$. Meanwhile, the strain amplitude was set from 0.01 to $10 \%$ ramped logarithmically and the excitation frequency was fixed at $1 \mathrm{~Hz}$. During both frequency and strain swept tests, the samples were energized by different magnetic fields of $0,1 \mathrm{~A}, 2 \mathrm{~A}, 3 \mathrm{~A}$, and $4 \mathrm{~A}$, respectively.

\section{RESULTS AND DISCUSSION}

\section{Morphological Appearances}

The morphologies of the cross-sectional MREs with a different weight fraction of CI particles are depicted in Figure 2. A random dispersion of CI particles within the MREs matrix is the primary requirement of the consistent performance in their functional

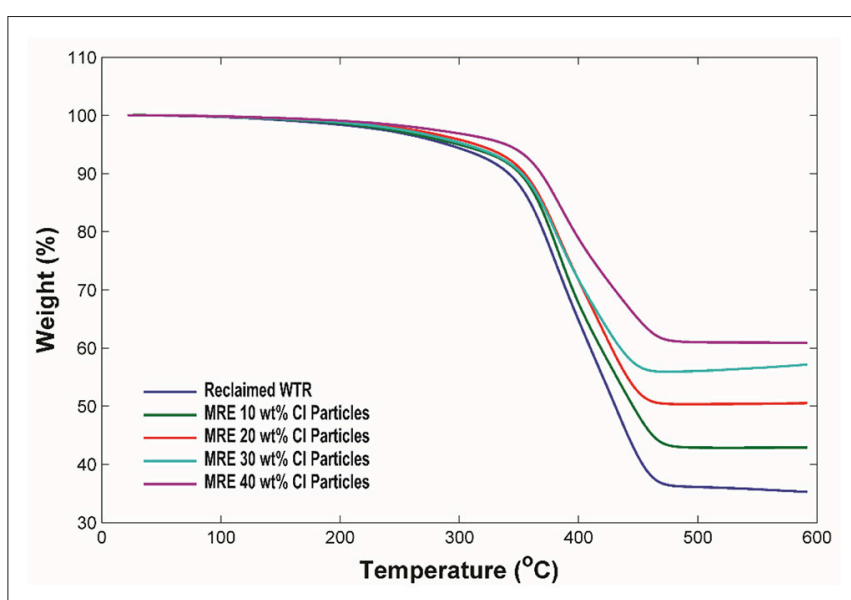

FIGURE 3 | Thermogravimetric curves of WTR based MREs.

TABLE 4 | Residual mass of decomposed WTR based MREs.

\begin{tabular}{lc}
\hline Sample & Residue $(\%)$ \\
\hline MREs 10 wt\% Cl particle & 61.08 \\
MREs 20 wt\% Cl particle & 55.95 \\
MREs 30 wt\% Cl particle & 50.46 \\
MREs 40 wt\% Cl particle & 42.93 \\
Reclaimed WTR & 36.23
\end{tabular}


behavior. A random distribution will ensure the magnetic flux to flow uniformly through the MREs. The dispersion of particles can be clearly observed in the SEM micrograph shown in Figure 2. The random distribution type of the MREs is confirmed by nonaligned dispersion of particles in the observed area. It can be seen that the CI particles adhere completely in the matrix. The weight percentage of each sample can be visually differentiated from the micrographs. The MREs with higher weight fraction of CI particles seem to be a closer distance and tend to agglomerate.

According to Figure 2, the micrograph of WTR based MRE confirmed that the fractured samples show well-blended rubber matrix. This fact indicates that the WTR has mixed thoroughly with each other as the results of high-pressure and hightemperature treatment. The blending of WTR can be considered as the occurrence of the revulcanization process. If the WTR only adhered to each other without curing, the matrix appearance of the fractured sample would exhibit different patterns. It would definitely show the WTR borders as the effect of non-blended WTR. Besides, the cryogenic effect during sample preparation would easily break the WTR interfaces when the rubber was not cured. The curing reaction during the reclamation process does not leave any particle boundaries among WTR.

\section{Swelling Properties}

A common issue that occurs in WTR reclamation is a revulcanization (crosslinking) achievement because the WTR belongs to inert materials. Therefore, the swelling test is often used to facilitate the evaluation of crosslinking of waste rubber reclamation. In this study, the degree of vulcanization of the MRE samples was also assessed by a swelling test to confirm the success of the revulcanization. The swelling properties including the soluble fraction, the swelling degree, the crosslinking density, and the degree of reclamation are listed in Table 3. Based on the calculation, the values are comparable to that of waste rubber reclamation through either a twin extruder or roll mills (Yazdani et al., 2011; Formela et al., 2014). For comparison purposes, Table 3 also provides the summary data of swelling properties obtained from the previous work. The values of the soluble

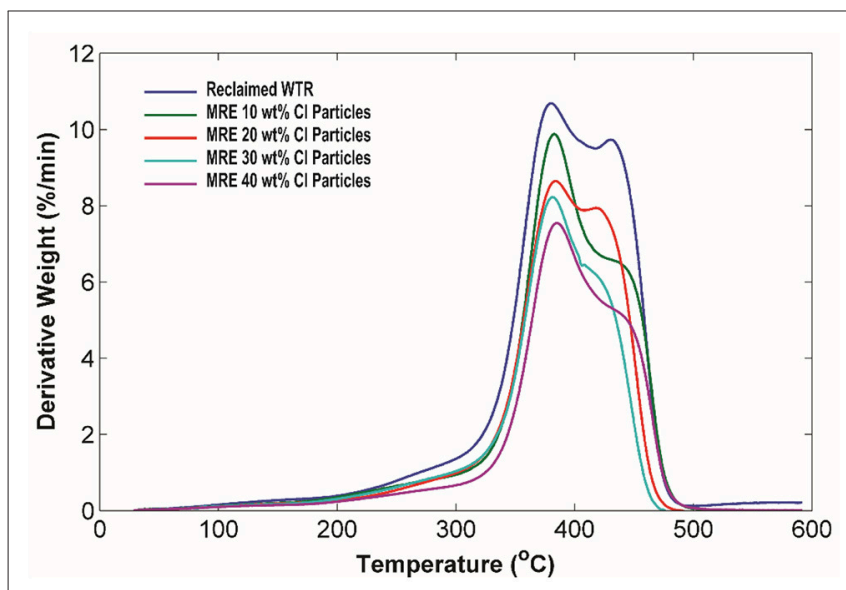

FIGURE 4 | DTG curves for pure reclaimed WTR and MREs. fraction and the swelling degree were decreased with the increase of CI particles. Moreover, the revulcanized MREs consists of crosslinked and free-chain portions. The immersion in toluene extracted the free chains. Meanwhile, the crosslinked sections were insoluble and swollen. The crosslink density values and degree of reclamation are in agreement with previous research reported by Formela et al. (2014). The degree of reclamation data was calculated and compared with the WTR without CI particles, and the results confirmed that the revulcanization was positive for the inert rubber during the thermomechanical reclamation.

A physical explanation of the successful mechanism of revulcanization can refer to some existing theories that has been proven through experimental works. Tobolsky et al. (1944), MacKnight et al. (1965) and Kende et al. (1965) initially discussed the theory of rubber reclamation. They found that the sulfursulfur bonds were the weakest chemical bonds with bond energies as follows: monosulfide crosslink (50-60 kcal/mol), disulfide crosslink (35 kcal/mol), and polysulfide crosslink $(27 \mathrm{kcal} / \mathrm{mol})$. Employing heat and pressure in certain degrees cleaved the sulfide linkages in the vulcanized rubber in situ during molding (Tripathy et al., 2002). Furthermore, Tobolsky (1960a,b) stated that when the polymers were protected from oxygen and other compounds at elevated temperatures, the rates of bond scission and reformation were principally equivalent. Tamura et al. (1983) also found that the secondary carbon atoms adjacent to the crosslinked moiety can be degraded when the isoprene rubber is heated beyond $200^{\circ} \mathrm{C}$. Therefore, based on the aforementioned reclamation theory, it is expected that the HPHT sintering would be sufficient for the second vulcanization of dead (?) rubber. During the fabrication of the MREs, the mixture of WTR, additives, and CI particles was compressed under high pressure. The high pressure could assist the removal of excessive voids. The absence of void during application of high pressure was confirmed by Tripathy et al. (2002) and Morin et al. (2002) who have implemented the HPHT reclamation technique for waste tire rubber.

\section{Thermal Analysis}

\section{Thermogravimetric Analysis}

Boczkowska et al. (2006) have initially carried out thermogravimetric analysis on MREs. The thermogravimetric test as part of thermal analysis can provide important material properties related to the stability of MREs against external temperature by identifying the thermal stability, oxidative mass reduction, and their decomposition performance. Boczkowska et al. (2006) reported that the additional iron particles to the elastomeric matrix would augment the thermal stability indicated from the lower oxidative mass loss at about $600^{\circ} \mathrm{C}$. The higher magnetizable particles within MRE samples, which consequently increase the thermal conductivity would have a better thermal conductivity. This is because the closer inter-particle distance is achieved as the higher fraction of magnetizable particles. For that reason, the MREs elements with a higher amount of iron particles would ease thermal energy flows across the samples.

MREs that use WTRs as the primary matrix would be facing issues related to thermal stability, which is critical to durability and age resistance. Thermogravimetric Analysis (TGA) can 


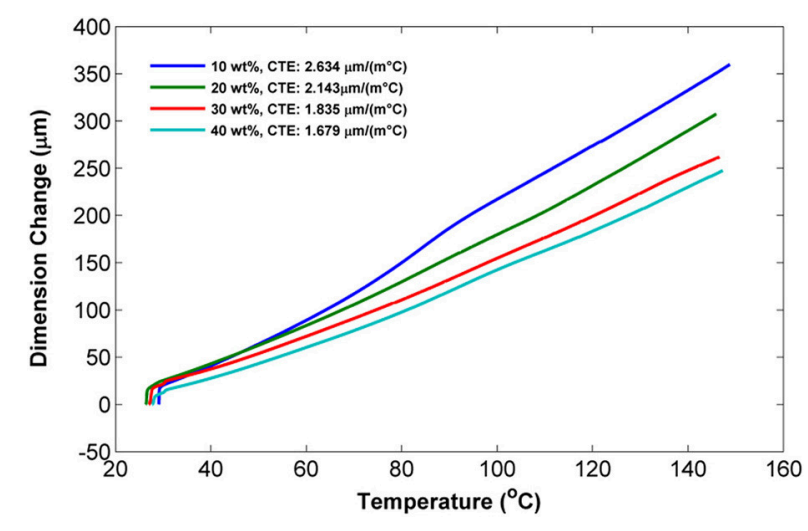

FIGURE 5 | Coefficient of thermal expansion curves of WTR based MREs.

be used to evaluate the thermal stability of a polymer. It can also be used to observe the content of compounds such as carbon black and ash in rubber. Figures 2, 3 show the TGA thermogram and corresponding derivative plots (DTG) of reclaimed WTR and MRE samples, respectively. As shown in Figure 3, the rubber content of all samples was entirely decomposed. The onset temperature of the volatile compounds released in all samples was $>200^{\circ} \mathrm{C}$. The volatile contents of the samples were determined at about from 5.15 to $9.85 \%$. The temperature, at which $5 \%$ (T5) mass loss happen, increases from $288.6^{\circ} \mathrm{C}$ (reclaimed WTR) to $341.9^{\circ} \mathrm{C}$ (MRE $40 \mathrm{wt} \% \mathrm{CI}$ particle). After that, the second transformation occurred involving the decomposition of rubber content that was releasing hydrocarbon compounds. During second transformation, there were changing slope values at temperature ranges of 379 to $385^{\circ} \mathrm{C}$. Furthermore, the second transformation has been stopped at a temperature that is higher than $450^{\circ} \mathrm{C}$. The final curves were maintained at the same weight until the next transformations. The changes of carbon content and CI particles occurred at temperatures higher than $600^{\circ} \mathrm{C}$. A weight reduction would either confirm the substance of the rubber or reflect the composition of CI particles carried by the rubber. A lower content of CI particles implies a higher weight reduction of the samples. In this study, the weight reductions of each sample until the end of the second transformation were evaluated by $33.77,36.88,41.31,47.99$, and 53.92 percent for MRE with 40,30, 20, 10, and 0 (pure rubber) wt\% of CI particles, respectively. The residual elements (at $600^{\circ} \mathrm{C}$ ) as detailed in Table 4 were carbon black and CI particles. Wang et al. (2006) found the same patterns in different CI particle compositions in MREs and concluded that the MRE with higher CI particle content exhibits better thermal stability.

At the second transformation, there was a slightly different slope that indicated the existence of overlapping thermal degradations. The different slope was also marked by the appearance of two peaks shown in the DTG curves in Figure 4. All samples shared a trend wherein the peaks were related to the maximum rate of thermal degradation temperature. The first peak was well identified and narrower than the second one. The sharp peak (first) indicated a deterioration of the natural

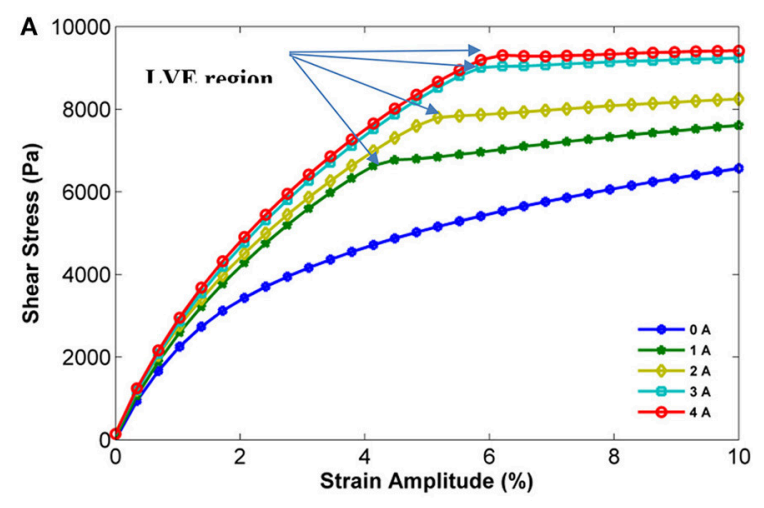

B

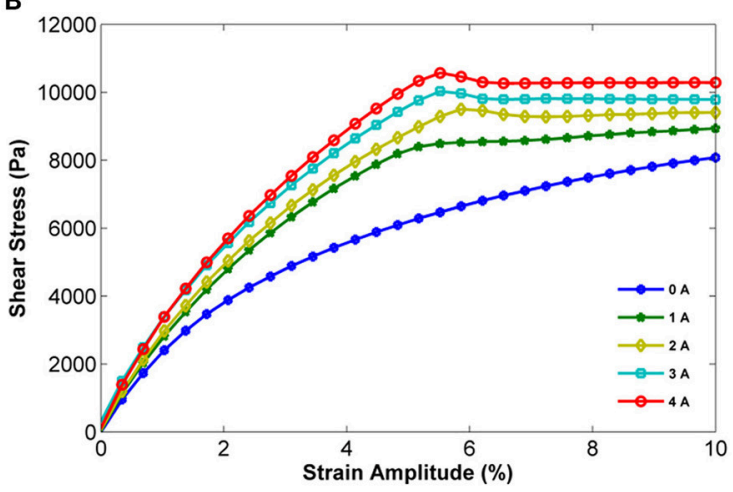

C

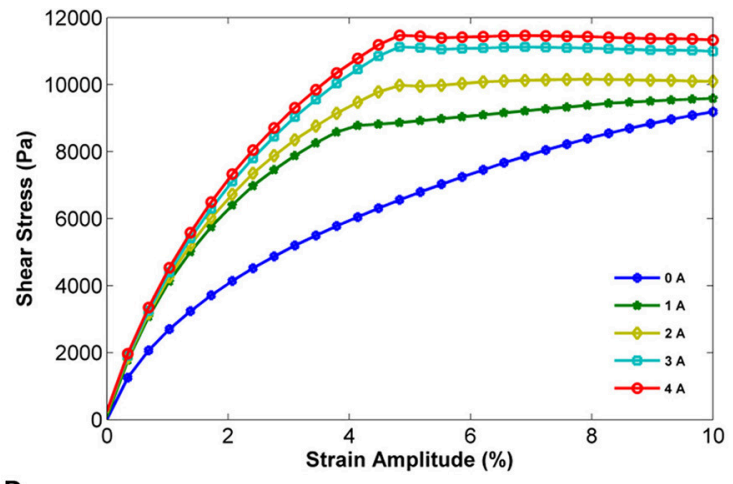

D

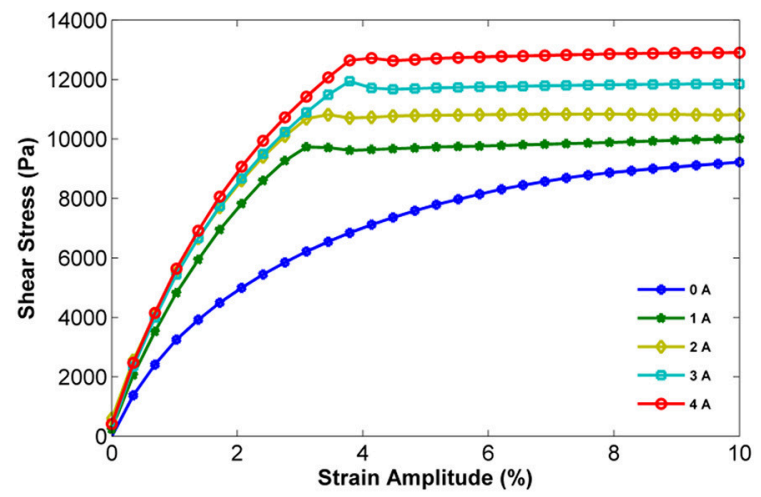

FIGURE 6 | Stress-strain curves of the MREs: (A) 10 wt\%, (B) 20 wt\%, (C) 30 $w t \%$, and (D) $40 \mathrm{wt} \%$. 
rubber (NR) at temperatures that ranged from 379.7 to $384.8^{\circ} \mathrm{C}$. By comparison, the temperature range for the broad peak was in between 418.6 and $449.3^{\circ} \mathrm{C}$. The temperature range for the second peak designated a synthetic rubber, which was styrenebutadiene rubber (SBR) (De and De, 2011). The mass loss rate decreased with an increase of CI particle content within the MREs. The values of peak mass loss rate at a temperature range of 379.7 to $384.8^{\circ} \mathrm{C}$ decreased from $10.68 \% / \mathrm{min}$ (reclaimed WTR) to $7.54 \% / \mathrm{min}$ (MRE $40 \mathrm{wt} \% \mathrm{CI}$ particle). Comparing the decomposition results with other studies, different authors (Kim et al., 1995; Gisbert et al., 2007) have reported similar results. Gisbert et al. (2007) found that the range of temperature for the decomposition of natural rubber was between 325.27 and $376.06^{\circ} \mathrm{C}$, while Kim et al. (1995) discovered the first peak at $377^{\circ} \mathrm{C}$. Moreover, the second broad peaks were reported to be in the range between 461.6 and $463.9^{\circ} \mathrm{C}$ by Gisbert et al. (2007), and $444^{\circ} \mathrm{C}$ by Kim et al. (1995). Based on the reported data, the TG/DTG results agreed with the fact that the tire rubber was a blend of various types of rubbers including both natural and synthetic rubbers.

\section{Thermogravimetric Analysis}

Thermal expansion is an important property for composite materials, particularly MREs. The test can reveal the behavior of material expansion during temperature alterations. For instance, the further application of MREs is common as a vibration insulator. The MREs must be bound well to a mounting bracket that is usually made of metals. When the mounted MREs work at engine temperature, a micro-scale expansion occurs at the mounting interface (between MREs and the mounting bracket). Since the coefficient of thermal expansion of the MREs portion is different from that of the steel bracket, thermal stress can occur at the MREs-steel bonding interface. Therefore, characterization of thermal expansion characteristic on MREs is often important to prevent product malfunction. The CTE evaluation of MREs is seldom reported. As far as could be ascertained, the thermal expansion of MREs has been reported by Nayak et al. (2015), which delivered the CTE of MREs based on silicon RTV.

Figure 5 depicts the dimension change of the MRE samples with increases in temperature. The expansion data of the MREs were taken under off-state conditions (no magnetic fields). The MRE samples changed their dimensions (micron size) in a positive slope, which resulted in an enlarged sample. The slopes decrease from 2.634 to $1.679 \mu \mathrm{m} /\left(\mathrm{m}^{\circ} \mathrm{C}\right)$ for MRE 10 to $40 \mathrm{wt} \%$ CI particle. Apparently, the higher slope was represented by the lower CI particle fraction contained in the MRE matrix. The

TABLE 5 | Linear viscoelastic region limit.

\begin{tabular}{lccccr}
\hline MREs sample & \multicolumn{5}{c}{ Strain (\%) } \\
\cline { 2 - 6 } & 0 A & 1 A & 2 A & 3 A & 4 A \\
\hline MRE 10 wt\% Cl particle & $>>10$ & 4.48 & 5.17 & 5.86 & 6.21 \\
MRE 20 wt\% Cl particle & $>>10$ & 5.17 & 5.86 & 5.52 & 5.52 \\
MRE 30 wt\% Cl particle & $>>10$ & 4.14 & 4.83 & 4.83 & 4.83 \\
MRE 40 wt\% Cl particle & $>>10$ & 3.1 & 3.45 & 3.79 & 3.79
\end{tabular}

smaller quantity of the slope implied that the sample with the higher content of CI particles has the lower expansion rate at an increase in temperature. This phenomenon is caused by the CTE of the CI particles, which is much lower than that of the rubber. The CTE of MR reported in Ubaidillah et al. (2016a) also depends on the state of the matrix. A softer matrix, such as silicon-based MREs, could produce a higher CTE, as reported by Nayak et al. (2015).

\section{Steady State Properties}

MREs have been acknowledged for their tuning capability of viscoelastic properties under external magnetic fields treatment. The rheological property such as shear modulus has a directly proportional correlation with the flux density. Therefore, the magnetic field dependent properties are a most important parameter of MREs. The stress-strain relationship of the waste tire based MREs was evaluated under steady state rotary shear at elevated current from 0 to $4 \mathrm{~A}$ sequentially and fix ambient temperature. This kind of test was relatively less studied since only minor information could be revealed such as shear modulus and the linear region at the very low strain. Previous publications on shear steady state properties of MREs can be found elsewhere (Tian et al., 2011; Qiao et al., 2012; Li and Nakano, 2013; Zhu et al., 2013; Mordina et al., 2014).

Figure 6 show the stress strain relationship under various magnetic fields. The shear rate was set to be $5 \mathrm{rad} / \mathrm{s}$ from 0 to $10 \%$ ramp linear strain. In off state condition, the stress grew up slowly until nearly reaching a constant value at $>10 \%$ strain. The curves exhibit different trends with on state condition. The stress growth in the presence of magnetic fields was faster than before and had slight overshoot before reaching steady stress. Based on the figures, all MREs types express an appreciable response to the presence of magnetic fields. The behaviors reflect the shear modulus enhancement when the magnetic fields increase from 0 to $3 \mathrm{~A}$. The increment of shear modulus appears as a steep rise at the current elevation, while, the increment upper than $3 \mathrm{~A}$ applied current is relatively small. The responsiveness of the MREs to the presence of magnetic fields increases from the lower iron particle fraction to the higher one. The almost linear increment of stress growth under magnetic fields confirmed the particle attraction. With these interactions, MREs behave as a solid material under a small strain. MREs exhibit a linear dependence on strain elevation until a specified strain limit is reached. Therefore, the region in which the shear modulus behaves in a linear condition is often referred to as the LVE region. Under a larger strain, the magnetized MREs exhibit a highly non-linear plastic flow in which the rigid chainlike structure of CI particles experience slippages between the particles and the reclaimed rubber matrix. At this stage, the stress overshoot appears to be significantly influenced by the shear orientation of the attracting particles. Another point of view about overshoot at the yield point is that the static friction caused by the surface roughness of MRE samples and a moving plate, which is a normal force, may contribute to the ultimate yield stress (Mordina et al., 2014).

The LVE regions of MRE based on Figure 6, under different magnetic fields regarding strain percentage, are detailed in 


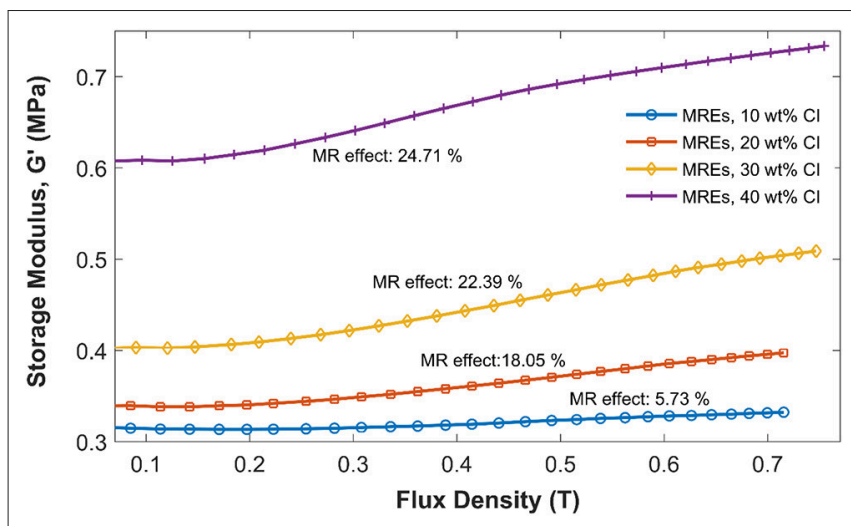

FIGURE 7 | Storage modulus under increment of magnetic fields.

Table 5. The limits of the linear region under magnetized conditions were influenced by CI particles composition and magnetic field density. Based on the stress-strain relationship, there was no agreement that either CI particles composition or magnetic field density have a dominant influence on the limit of LVE region. Based on Table 5 as well as Figure 6, the higher CI particle content was followed by the smaller LVE region except for MRE $20 \mathrm{wt} \%$ at 1 and $2 \mathrm{~A}$ tests. These 2 points were considered as data uncertainties since the other test condition showed normal trends. Moreover, the higher field density also slightly changed the LVE region. As a highlight, the attractive force among particles was one of the determining factors of the LVE region, and was greatly influenced by both the CI particles fraction and the magnetic field density.

\section{Dynamic Properties}

Figure 7 portrays the change of storage modulus in each sample as a function of the magnetic flux. While the magnetic flux is raised up, all specimens exhibit the augmentations of storage modulus in different quantities. The MREs with small contents of CI particles exhibit a slight modulus growth associated with those having the big fraction. The magnetic fields cause an interparticle attraction forming a strong magnetic reinforcement. The elevated modulus is an implication of the aforementioned phenomenon. The fractional difference called MR effect between on state $(5 \mathrm{~A})$ and off state $(0 \mathrm{~A})$ is also shown in Figure 7. The highest MR effect was achieved by the sample with $40 \mathrm{wt} \% \mathrm{CI}$ particles that is $24.71 \%$.

Furthermore, this sample was tested to achieve dynamic properties under increment of excitation frequency and strain amplitude. Figures 8A-C display the trends of respective storage modulus, loss modulus, and loss factor under increment of excitation frequency. Both storage and loss moduli demonstrated the same behavior: this being augmentation of each value when the magnetic flux was amplified. The phenomenon of increased storage modulus as an effect of frequency suggests that at a higher excitation frequency, the MR elastomer samples have a larger storage modulus. When the different current was applied, it also affected the increment of the storage modulus at a certain frequency. Moreover, the magneto-induced loss
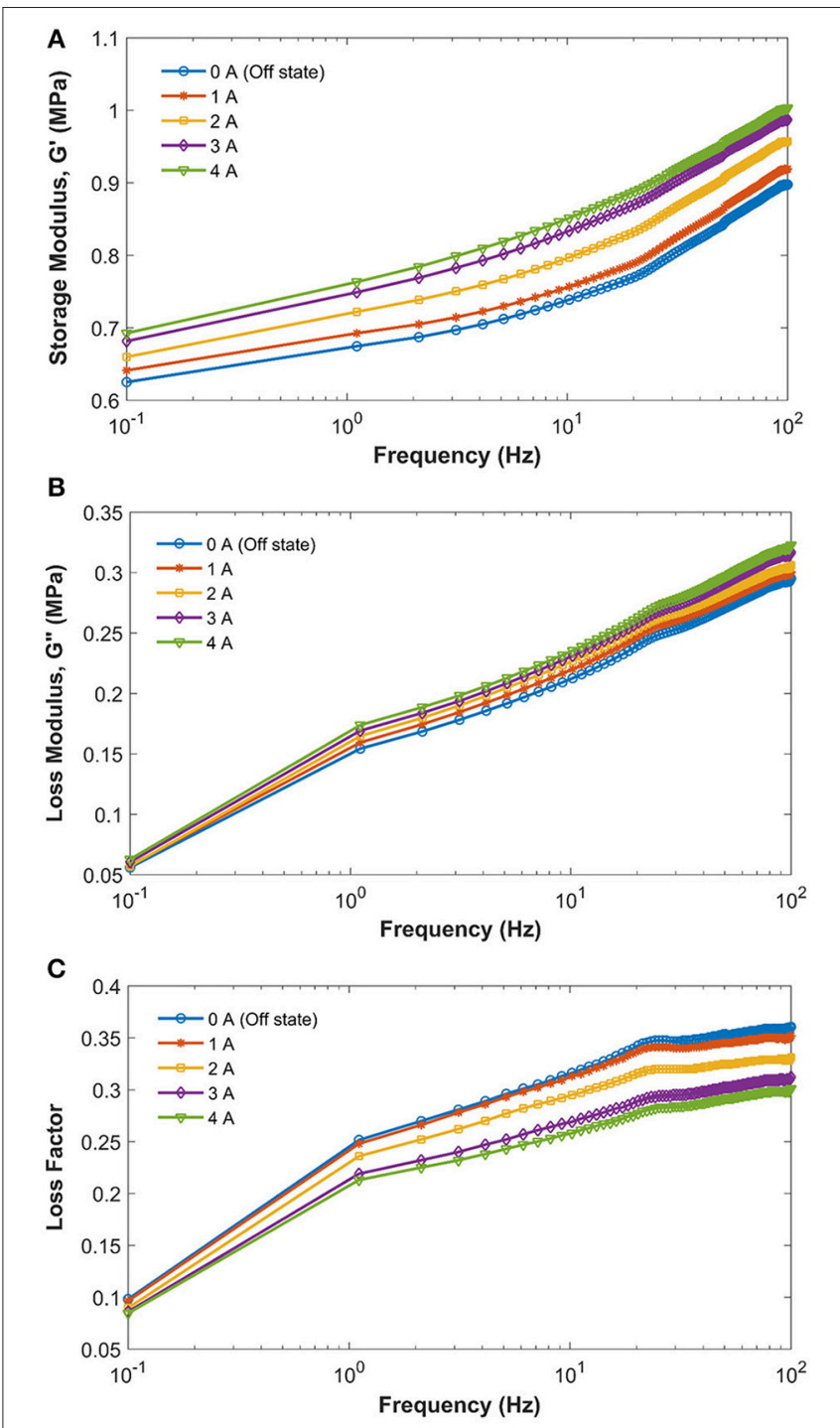

FIGURE 8 | Dynamic properties of WTR based MREs with 40 wt\% CIP under frequency domain. (A) Storage Modulus, (B) Loss Modulus, and (C) Loss Factor.

modulus was increased monotonically with the frequency for all measured variants in both off- and on-states. In the offstate, the filler particles of both iron particles and carbon black served to strengthen the matrix when subjected to a higher frequency. The enhancement of the loss modulus reflected how the MR elastomer materials dissipate more energy at higher frequencies. Different to storage and loss moduli, the loss factor which represents phase-lag between stress and strain, exhibits a decrement value when the sample was subjected to the increase of magnetic flux. The magnetic flux strongly affects the inter-particle attraction forces. Thus, the relative motion between CI particles is clogged resulting in the reduction of slipping displacement of the other fillers such as carbon black.

Figures 9A-C show the behavior of WTR based MREs under ramped strain amplitude. The storage moduli diminished in the initial regions and underwent drastic drops up to a $10 \%$ 

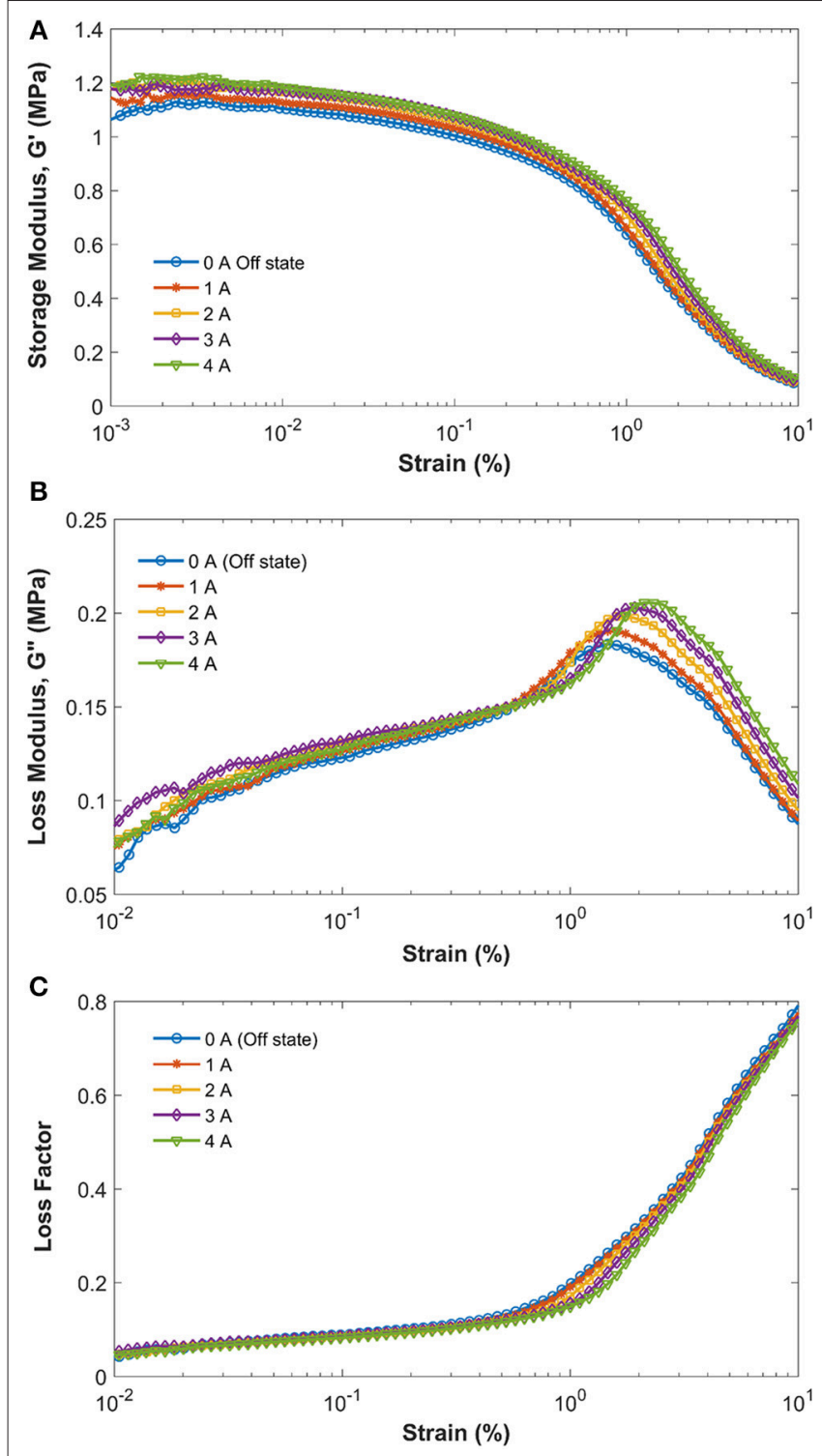

FIGURE 9 | Dynamic properties of WTR based MREs with 40 wt\% CIP under strain domain: (A) Storage modulus, (B) Loss modulus, and (C) Loss factor.

strain amplitude. As can be seen in Figure 9A, the Payne effect appears in both on and off states. The strain dependence modulus in the off-state is purely influenced by filler-filler and filler-matrix interactions. Meanwhile, during on state situation the magnetic attraction strengthens the filler-fillermatrix interactions. Therefore, the magnetic attraction between CI particles escalates the storage modulus as the increase of magnetic flux. The same trends went to the strain dependence loss modulus as depicted in Figure 9B. The increase of magnetic fields also augmented the loss modulus values. However, the alteration was not clearly indicated at a lower strain amplitude. At the strain where the storage modulus dropped caused by Payne Effect, the loss modulus also exhibited the same situation. The loss modulus increased from low to medium strain followed by dramatic drops at the corresponding strain until 10\%. As stated previously, the increase of magnetic flux reduced the phase-lag between stress and strain. Therefore, the loss factor also diminished at the time increasing magnetic flux and strain amplitude as can be seen in Figure 9C. However, the decrement of loss factor under ramped strain was not clearly determined compared to those under ramped frequency (refer to Figure $\mathbf{8 C}$ ).

\section{CONCLUSION}

In this work, the morphology, swelling properties, thermal analysis, and rheological tests of the WTR based MREs have been investigated to ensure the reclamation achievement and thermal stability. The three-dimensional cross-link of inert rubber has been successfully reformed for the MREs matrix through HPHT sintering process. This phenomenon was confirmed by the swelling tests for the MREs, in which the degree of reclaiming achieved was higher than $54 \%$. The weight reduction trends have exhibited that a logical pattern is proven in which the lower filler content was accompanied by a higher weight reduction percentage compared to the high iron particle fraction. The TMA results confirmed that the lower rubber content led to the lower coefficient of thermal expansion. The steady state test confirmed that the linear viscoelastic were about $3 \%$ to $6.5 \%$ strain in on state condition. The dynamic tests of the samples were also studied to justify rheological behavior under oscillating loads. Under increment of the magnetic flux, the storage moduli were augmented and showed the maximum MR effect of $24.71 \%$. It is finally remarked that the results presented in this work can be referred as useful data to demonstrate high performance of smart MREs based on the waste tire rubber.

\section{DATA AVAILABILITY}

The datasets generated for this study are available on request to the corresponding author.

\section{AUTHOR CONTRIBUTIONS}

$\mathrm{U}$ : experiment, writing, and formatting; EP: writing and formatting; HI, S-BC, AA, and SM: writing and editing.

\section{FUNDING}

This research has been supported by Mandatory Research Grant, 2019, UNS and USAID through Sustainable Higher Education Research Alliances (SHERA) Program-Centre for Collaborative (CCR) National Center for Sustainable Transportation Technology (NCSTT) with Contract No. IIE00000078-ITB-1.

\section{ACKNOWLEDGMENTS}

This work is supported by Universitas Sebelas Maret through Mandatory Research Grants 2019. Authors thank to Universiti Teknologi Malaysia (UTM) for research facilities. 


\section{REFERENCES}

Ausanio, G., Iannotti, V., and Ricciardi, E. (2014). Magneto-piezoresistance in Magnetorheological elastomers for magnetic induction gradient or position sensors. Sensors Actuators A: Phys. 205, 235-239. doi: 10.1016/j.sna.2013.10.009

Bica, I., Anitas, E. M., and Bunoiu, M. (2014). Hybrid magnetorheological elastomer: influence of magnetic field and compression pressure on its electrical conductivity. J Indus Eng Chem. 20, 3994-3999. doi: 10.1016/j.jiec.2013.12.102

Boczkowska, A., Awietjan, S. F., and Babski, K. (2006). "Effect of the processing conditions on the microstructure of urethane magnetorheological elastomers," in Smart Structures and Materials: Active Materials: Behavior and Mechanics. ed W. D. Armstrong (San Diego, CA: SPIE Digital Library), 61700R-61700R11. doi: 10.1117/12.651668

De, D., and De, D. (2011). Processing and material characteristics of a reclaimed ground rubber tire reinforced styrene butadiene rubber. Mater. Sci. Appl. 2, 486-495. doi: 10.4236/msa.2011.25066

De, D., Panda, P. K., and Roy, M. (2013). Reinforcing effect of reclaim rubber on natural rubber/polybutadiene rubber blends. Mater. Design 46, 142-150. doi: $10.1016 /$ j.matdes.2012.10.014

Flory, P. J., and Rehner, J. (1943). Statistical mechanics of cross-linked polymer networks I. Swelling. J. Chem. Phys. 11:521. doi: 10.1063/1.1723792

Formela, K., Cysewska, M., and Haponiuk, J. (2014). The influence of screw configuration and screw speed of co-rotating twin screw extruder on the properties of products obtained by thermomechanical reclaiming of ground tire rubber. Polimery 59, 170-177. doi: 10.14314/polimery.2014.170

Ghafoorianfar, N., Wang, X. J., and Gordaninejad, F. (2013). "On the sensing of magnetorheological elastomers," in Sensors and Smart Structures Technologies for Civil, Mechanical and Aerospace System. eds J. P. Lynch, C.-B. Yun, and K.-W. Wang, 869214-1-869214-10. doi: 10.1117/12.2009944

Gisbert, A. N., Crespo, A. J. E., and López, M. J. (2007). Study of thermal degradation kinetics of elastomeric powder (ground tire rubber). Polym. Plast. Technol. Eng. 47, 36-39. doi: 10.1080/03602550701580870

Kende, I., Pickering, T. L., and Tobolsky, A. V. (1965). The dissociation energy of the tetrasulfide linkage. J. Am. Chem. Soc. 87, 5582-5586. doi: 10.1021/ja00952a012

Kim, S., Park, J. K., and Chun, H. D. (1995). Pyrolysis kinetics of scrap tire rubbers. I: using DTG and TGA. J. Env. Eng. 121, 507-514. doi: 10.1061/(ASCE)0733-9372(1995)121:7(507)

Li, W. H., Kostidis, K., and Zhou, Y. (2009). "Development of a force sensor working with MR elastomers," in International Conference on Advanced Intelligent Mechatronics (Singapore: IEEE), 233-238. doi: 10.1109/AIM.2009.5230010

Li, W. H., and Nakano, M. (2013). Fabrication and characterization of PDMS based magnetorheological elastomers. Smart Mater. Struct. 22:55035. doi: 10.1088/0964-1726/22/5/055035

Li, Y. C., Li, J. C., and Li, W. H. (2014). A state-of-the-art review on magnetorheological elastomer devices. Smart Mater. Struct. 23:123001. doi: 10.1088/0964-1726/23/12/123001

MacKnight, W. J., Leroi, G. E., and Tobolsky, A. V. (1965). Physical chemistry of crosslinked polysulfide elastomers. J. Chem. Educ. 42, 1-7. doi: 10.1021/ed042p4

Mordina, B., Tiwari, R. K. R. K., and Setua, D. K. D. K. (2014). Magnetorheology of polydimethylsiloxane elastomer/FeCo 3 nanocomposite. J. Phys. Chem. C 118, 25684-25703. doi: 10.1021/jp507005s

Morin, J. E., Williams, D. E., and Farris, R. J. (2002). A novel method to recycle scrap tires: high-pressure high-temperature sintering. Rubber Chem. Technol. 75, 955-968. doi: 10.5254/1.3547695

Nayak, B., Dwivedy, S. K. K., and Murthy, K. S. S. (2015). Fabrication and characterization of magnetorheological elastomer with carbon black. J. Intell. Mater. Syst. Struct. 26, 830-839. doi: 10.1177/1045389X14535011

Ottaviani, R. A., Ulincy, J. C., and Golden, M. A. (2005). Magnetorheological Nanocomposite Elastomer for Releasable Attachment Applications.

Piszczyk, Ł., Hejna, A., and Formela, K. (2014). Effect of ground tire rubber on structural, mechanical and thermal properties of flexible polyurethane foams. Iranian Polymer J. 24, 75-84. doi: 10.1007/s13726-014-0301-4
Qiao, X. Y., Lu, X. S., and Li, W. H. (2012). Microstructure and magnetorheological properties of the thermoplastic magnetorheological elastomer composites containing modified carbonyl iron particles and poly(styrene- b -ethyleneethylenepropylene- b -styrene) matrix. Smart Mater. Struct. 21:115028. doi: 10.1088/0964-1726/21/11/115028

Sorokin, V. V., Stepanov, G. V., and Shamonin, M. (2015). Hysteresis of the viscoelastic properties and the normal force in magnetically and mechanically soft magnetoactive elastomers: effects of filler composition, strain amplitude and magnetic field. Polymer. 76, 191-202. doi: 10.1016/j.polymer.2015.08.040

Tamura, S., Murakami, K., and Kuwazoe, H. (1983). Isothermal degradation of cis-1,4-polyisoprene vulcanizates. J. Appl. Polymer Sci. 28, 3467-3484. doi: 10.1002/app.1983.070281112

Tian, T. F., Li, W. H., and Alici, G. (2011). Microstructure and magnetorheology of graphite-based MR elastomers. Rheologica Acta 50, 825-836. doi: 10.1007/s00397-011-0567-9

Tobolsky, A. V. (1960a). Polymer Science and Materials. New York, NY: Interscience.

Tobolsky, A. V. (1960b). Properties and Structures of Polymers. New York, NY: John Wiley and Sons, Inc.

Tobolsky, A. V., Prettyman, I. B., and Dillon, J. H. (1944). Stress relaxation of natural and synthetic rubber stocks. J. Appl. Phys. 15:380. doi: 10.1063/1.1707442

Tripathy, A. R., Morin, J. E., and Williams, D. E. (2002). A novel approach to improving the mechanical properties in recycled vulcanized natural rubber and its mechanism. Macromolecules 35, 4616-4627. doi: 10.1021/ma012110b

Ubaidillah, C., Jin Choi, H., Amri Mazlan, S., and Imaduddin, F. (2016a). Fabrication and viscoelastic characteristics of waste tire rubber based magnetorheological elastomer. Smart Mater. Struct. 25, 1-14. doi: 10.1088/0964-1726/25/11/115026

Ubaidillah, S., Amri Mazlan, S., Sutrisno, J., and Yahya, I. (2016c). Physicochemical properties and stress-strain compression behaviors of a ground tire rubber based magnetorheological elastomers. Scientia Iranica, Trans. C: Chem. Chem. Eng. 23, 1144-1159. doi: 10.24200/sci.2016.3885

Ubaidillah, S., Imaduddin, F., Li, S., and Amri Mazlan, S. (2016b). A new class of magnetorheological elastomers based on waste tire rubber and the characterization of their properties. Smart Mater. Struct. 25, 1-15. doi: 10.1088/0964-1726/25/11/115002

Ubaidillah, S. J., Purwanto, A., and Amri Mazlan, S. (2015). Recent progress on magnetorheological solids: materials, fabrication, testing, and applications. Adv. Eng. Mater. 17, 563-597. doi: 10.1002/adem.201400258

Wang, Y. H., Zhang, X. R., and Oh, J. E. (2015). Fabrication and properties of magnetorheological elastomers based on CR/ENR self-crosslinking blends. Smart Mater. Struct. 24:95006. doi: 10.1088/0964-1726/24/9/ 095006

Wang, Y. L., Hu, Y., and Deng, H. X. (2006). Magnetorheological elastomers based on isobutylene-isoprene rubber. Polymer Eng. Sci. 46, 264-268. doi: 10.1002/pen.20462

Yazdani, H., Karrabi, M., and Ghasmi, I. (2011). Devulcanization of waste tires using a twin-screw extruder: the effects of processing conditions. J. Vinyl Addit. Technol. 17, 64-69. doi: 10.1002/vnl.20257

Zhu, J. T., Xu, Z. D., and Guo, Y. Q. (2013). Experimental and modeling study on magnetorheological elastomers with different matrices. J. Mater. Civil Eng. 25, 1762-1771. doi: 10.1061/(ASCE)MT.1943-5533.0000727

Conflict of Interest Statement: The authors declare that the research was conducted in the absence of any commercial or financial relationships that could be construed as a potential conflict of interest.

Copyright (c) 2019 Ubaidillah, Purnomo, Ismail, Choi, Aziz and Mazlan. This is an open-access article distributed under the terms of the Creative Commons Attribution License (CC BY). The use, distribution or reproduction in other forums is permitted, provided the original author(s) and the copyright owner(s) are credited and that the original publication in this journal is cited, in accordance with accepted academic practice. No use, distribution or reproduction is permitted which does not comply with these terms. 